\title{
Sustentabilidad y recurso hídrico: la agricultura en unidades campesinas en el departamento de Boyacá
}

\author{
Wilson González Santos \\ Universidad Pedagógica y Tecnológica de Colombia, Tunja \\ wilson.gonzalez@uptc.edu.co
}

\section{Resumen}

Ante los embates de la crisis social, ambiental, económica e institucional en nuestros etnoecosistemas, que ocasiona una alta entropía y un coste de residuos que amenazan seriamente las posibilidades de la biosfera y su resilencia, se plantea del desarrollo sustentable como un camino que permite opciones de crecimiento y de-crecimiento en función tanto de la calidad de vida del colectivo humano como del stock de recursos disponibles en nuestro planeta. Con el presente trabajo y con la aplicación de la metodología MESMIS se muestra como la oferta, el manejo y la persistencia del recurso hídrico en unidades productivas campesinas es una apuesta que obliga a repensar la sostenibilidad ambiental en términos de la relación integralidad-sustentabilidad y calidad de vida. Una mirada a los arreglos espaciales de la agricultura campesina en Boyacá permite comprender que el recurso hídrico hace parte de la integralidad de su etnoecosistema y que su preservación dista de miradas segmentadas. Los mapas multicriterio evidencian la necesaria inclusión de la sostenibilidad social como un puente para alcanzar la sostenibilidad ambiental.

Palabras Clave: Sustentabilidad, integral, desarrollo, crisis, persistencia.

\section{Sustainability and water resource: agriculture in peasant units in the department of Boyacá}

\section{Abstract}

Facing the battering of social, environmental, economic and institutional crisis in our ethnoecosystems, resulting in a high entropy and cost of waste that affect the biosphere and its resilience, sustainable development is a way that allows options for growth and decreased so much the quality life of the human collective as well as the stock of resources available on our planet. With this work and with the implementation of the MESMIS methodology is shown as the supply, management and persistence of water resources in peasant units is a bet that forces us to rethink environmental sustainability in terms of comprehensiveness-sustainability and quality relationship of life. A look at the spatial arrangements of Boyacá peasant agriculture allows us to understand that the water resource is part of the integrity of its etnoecosistema and that its preservation is far from segmented sights. Multicriteria maps show the necessary inclusion of social sustainability as a bridge to reach the environmental sustainability.

Keywords: Sustainability, development, crisis, persistence. 


\section{Introducción}

La persistencia de la agricultura campesina ha sido objeto de varias aproximaciones. Los estudios abarcan posiciones clásicas chayanovianas que explican su permanencia en función de las características de su unidad productiva. Para esta argumentación, una unidad campesina al ser a la vez - un espacio de producción y de consumo - con una orientación de mantenimiento y reproducción, trasciende lo puramente mercantil y busca la reproducción familiar campesina. En otro sentido, se encuentran los enfoques relacionados con la agricultura capitalista. Los seguidores de esta última orientación avizoraban la extinción de la agricultura campesina, dada su poca competitividad económica, que le impedía sobrevivir al avance del capitalismo y en consecuencia sus actores principales, los campesinos, terminaban absorbidos por éste convirtiéndose en proletariados rurales (Boltvinik, 2012; Mora, 2007).

De acuerdo con lo anterior y, aunque las dos posturas ofrecen elementos importantes para la comprensión de la agricultura campesina, omiten la perspectiva holística necesaria para el análisis de un grupo humano para quien la integralidad, la biodiversidad y la interacción con su nicho es el soporte de su sostenimiento. Al respecto, González (2009) admite la necesidad de la inclusión de la inter y transdisciplinariedad en los estudios sobre lo rural. En la misma línea, Pirazán (2016) en investigaciones realizadas en el departamento de Boyacá indica que para la entrada al estudio de la agricultura campesina, la complejidad puede ser el camino propicio. El autor en cuestión utiliza la sustentabilidad como una aproximación oportuna ya que incluye dicha perspectiva. Como se puede inferir, los autores alertan la necesidad de trascender la fragmentación y/o abstracción del cientificismo cartesiano para la comprensión de la ruralidad campesina.

Si bien es cierto el concepto de sustentabilidad ha tenido diversas aplicaciones, en la ejercicio en cuestión y de acuerdo con Olmos (2014) se aborda desde el análisis de la complejidad en una unidad campesina. El investigador subraya la hipótesis de que la sustentabilidad es una condición que permite explicar la subsistencia campesina. Aunado a lo anterior, concibe la sustentabilidad del recurso hídrico como una consecuencia de la sustentabilidad social. De esta manera la sustentabilidad social es el viaducto para alcanzar la sustentabilidad ecológica (Foladori y Tomassino, 2000).

El siguiente artículo hace una reflexión en torno a la agricultura campesina y su persistencia. Con base en estudios realizados en el departamento de Boyacá, Colombia, el autor muestra como la sustentabilidad, para la cual el recurso hídrico es soporte de su permanencia, es la perspectiva pertinente para explicar la persistencia de las unidades campesinas.

\section{Materiales y métodos}

La reflexión se hizo con base en estudios realizados en la zona centro y occidente del departamento de Boyacá, en especial en los municipios de Soraca, Muzo y Coper, Colombia. Se utilizó la investigación documental y el método teórico hipotético - deductivo, ya que a partir de unas premisas, se hacen inferencias que derivan en un resultado, que además se había pretendido demostrar (Hernández, 2008). El método empírico fue el Marco para la Evaluación de Sistemas de Manejo de Recursos Naturales incorporando Indicadores de Sustentabilidad. Las técnicas hacen parte del Diagnóstico Rural participativo y de la cartografía social en un muestreo dirigido no probabilístico ya que se hace énfasis en el estudio a profundidad de una unidad rural, la finca campesina. En este caso, la hipótesis señala que las unidades campesinas son sustentables y la sustentabilidad del recurso hídrico es consecuencia de una sustentabilidad social.

\section{Resultados y discusión}

La relación de la sustentabilidad y del recurso hídrico en unidades campesinas propone una detención en la manera de abordarlo. Si bien es cierto, el carácter integral, holístico y multidimensional de la sustentabilidad obligan a su mirada desde la perspectiva compleja, también lo es que su cuantificación es necesaria 
a fin de comprender tanto sus efectos como las posibilidades para lograr una intervención cercana al quehacer de los actores involucrados. De esta manera, dicha cuantificación permite transformar la complejidad de una naturaleza en en indicadores claros, objetivos y generales (Sarandón, 2002). La anterior situación se aclara si se tiene en cuenta que la abstracción, aunque limitante para la comprensión de la integralidad, es consecuente para la intervención antrópica.

Luego de la anterior consideración, las figuras 1 y 2 muestran los mapas multicriterios obtenidos luego de la aplicación de la metodología MESMIS (Marco para la Evaluación de Sistemas de Manejo de Recursos Naturales incorporando Indicadores de Sustentabilidad) que permite revelar, normalizar y ponderar las distintas relaciones existentes entre las diferentes dimensiones propias al concepto de sustentabilidad (Astier et al., 2008). En las figuras 1 y 2 , un acercamiento a los vértices del poligono denota una mayor sustentabilidad en los distintos valores. Nótese que los indicadores corresponden con las diferentes dimensiones propias de la sustentabilidad: social, ambiental y económica. En este sentido, se omite la abstracción que puede contener una perspectiva puamente ecológica. Al respecto, La Declaración de Dublín sobre el Agua y el Desarrollo Sostenible señala en su Principio №1 señala:

Since water sustains life, effective management of water resources demands a holistic approach, linking social and economic development with protection of natural ecosystems. Effective management links land and water uses across the whole of a catchment area or groundwater aquifer.

El investigador prefiere en principio el su texto en su lengua de origen dadas las diversas interpretaciones que se han usado en su traducción, No obstante, hace énfasis en dos aspectos vitales para la presente reflexión: la aproximación integral y en consecuencia, la necesaria relación que debe existir entre la protección del recurso hídrico y el desarrollo económico y social.

El recurso hidrico de las unidades campesinas de Muzo y Coper se fundamenta en arreglos espaciales biodiversos provistos de cobertura vegetal (nivel de sustentabilidad: 5). No obstante, la perpectiva de la sustentabilidad trasciende lo estrictamente ecológico. De esta manera, la provisión y mantenimiento del agua como soporte de las actividades agricolas y pecuarias de las unidades campesinas se concilia con aspectos socio ecopomicos como los evidenciados en los indicadores de integración social y acceso a la salud y educación (nivel de sustentabilidad: entre 4 y 5 ).

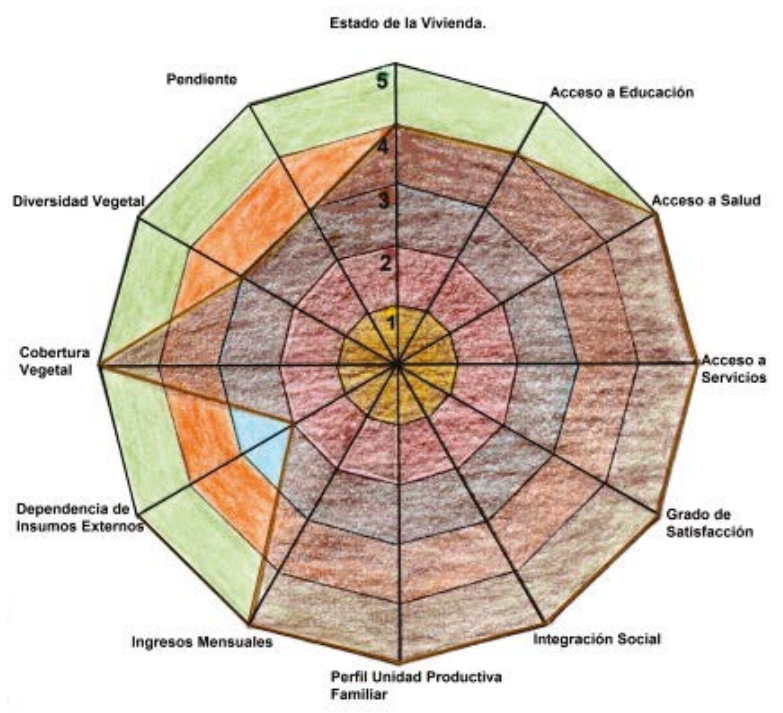

Figura 1. Mapa multicriterio de la unidad campesina cacaotera en Muzo, Boyacá.

Fuente: Olmos (2014)

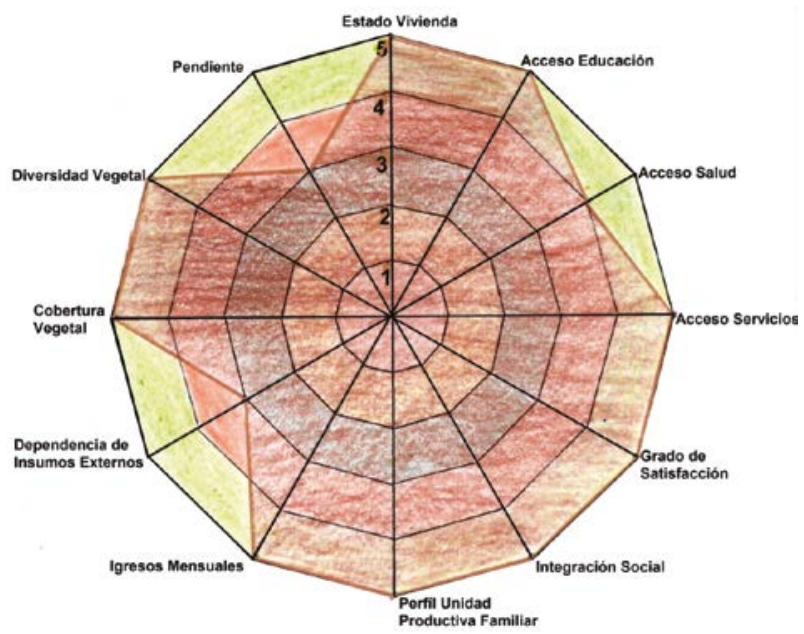

Figura 2. Mapa multicriterio de la unidad campesina cacaotera en Coper, Boyacá.

Fuente: Olmos (2014) 
Una mirada a los arreglos espaciales propios de los predios de unidades campesinas en el municipio de Soracá subraya la biodiversidad (por lo menos tres actividades agropecuarias distintas) (Figuras 3 y 4). Aunado a lo anterior, el autoconsumo va atado a este tipo de producción y es un criterio relevante para caracterizar la economía campesina. En concordancia, Chayanov (1974) indicaba que los campesinos se identifican por la participación activa de su economía familiar quien además produce para su propio consumo. La unidad campesina se enfoca en su autosustento alimenticio. Si se acepta esta premisa, se puede inferir que la multidimensionalidad inherente a la agricultura campesina es consecuente con su sustentabilidad. Con lo anterior, el recurso hídrico concierta con los demás componentes a fin de orientar la racionalidad campesina. En concordancia, la motivación de la unidad campesina trasciende lo puramente económico y se concentra en la supervivencia familiar cuyo soporte es la intervención ecuánime en los recursos que integran su unidad productiva.

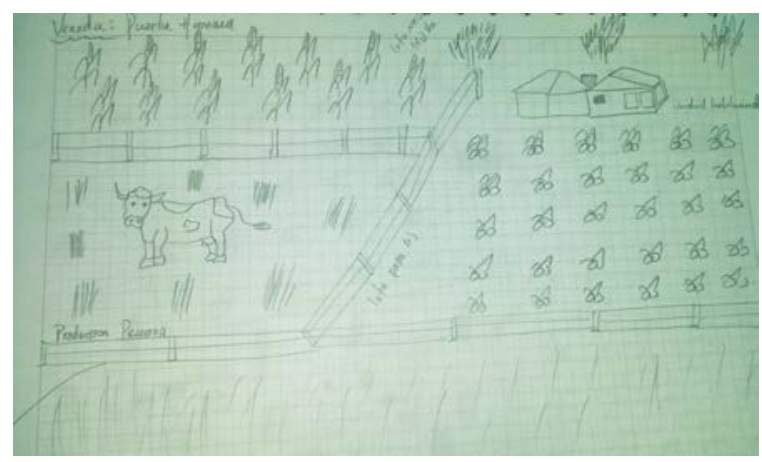

Figura 3. Finca tipo de la unidad campesina en Soraca. Fuente: Pirazan (2016)

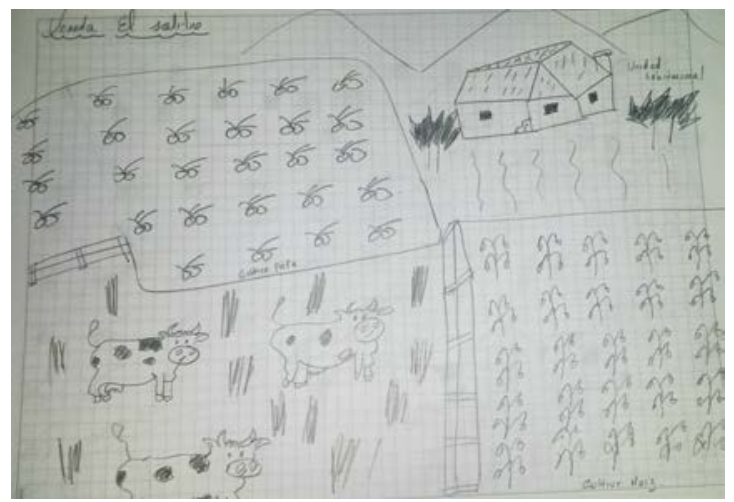

Figura 4. Finca tipo de la unidad campesina en Soraca. Fuente: Pirazan (2016)

\section{Conclusiones}

Las unidades campesinas del departamento de Boyacá mantienen su persistencia en función de arreglos espaciales biodiversos donde se concilian distintos componentes bajo la perspectiva de la sustentabilidad.

La sustentabilidad del recurso hídrico trasciende la mirada propia de la sustentabilidad ecológica. La investigación enfatiza que el abordaje pasa por el análisis holístico, los diseños multicriterios y la aproximación compleja, que se traduce en la sustentabilidad social.

\section{Referencias bibliográficas}

Astier, M., Masera., O., Yankuic, M., (2008). Evaluación de la sustentabilidad. Un enfoque dinámico y multidimenciomanal. Imag impresssions., Valencia, 200 pp.

Boltvinik, J., (2012). Pobreza y persistencia del campesinado. Mundo siglo XXI, 8(28), 1939.

Chayanov, A., (1974). Las concepciones de economia campesina. La organizacion de la unidad economica campesina. Ediciones nueva vision., Buenos Aires,

Foladori, G., Tommasino, H., (2000). El concepto de desarrollo sustentable 30 años después. Cuadernos de Desarrollo e Meio Ambiente. 4, 41-56.

González, W., (2009). Educación y entorno en la ingenieria agronómica. Universidad Pedagógica y Tecnológica de Colombia. Tunja, $60 \mathrm{pp}$.

Hernández, A., (2008). El método hipotéticodeductivo como legado del positivismo lógico y el racionalismo crítico: su influencia en la economía. Ciencias Económicas (26), 183195.

Mora, J., (2007). Persistencia, conocimiento local y estrategias de vida en sociedades campesinas. Revista Estudios sociales. 29, 122-133. 
Olmos, M. (2014). Evaluación de la sustentabilidad de unidades cacaoteras en explotaciones familiares del occidente de Boyacá. (Tesis de Maestría en Desarrollo Rural). Universidad Pedagógica y Tecnológica de Colombia. Tunja.

Pirazan, I. (2016). La producción agraria campesina en el municipio de Soracá (Boyacá) ¿cambios o adaptabilidad? Periodo 1985 - 2015. (Tesis de Maestría en Desarrollo Rural). Universidad Pedagógica y Tecnológica de Colombia. Tunja.

Sarandón, S., (2002). El Desarrollo y Uso de Indicadores para evaluar la sustentabilidad de los Agroecosistemas. Ediciones Científicas Americanas., La Plata, 414 pp. 